\title{
Toxic dilatation of the colon in shigellosis
}

\author{
A P R Wilson, G L Ridgway, M Sarner, P B Boulos, M G Brook, G C Cook
}

Toxic megacolon can result from shigellosis in travellers even when faecal culture is negative

\section{Department of \\ Microbiology, University College Hospital, London WC1E 6AU \\ A P R Wilson, MD, senior lecturer in microbiology \\ G L Ridgway, MD, consultant microbiologist \\ Department of Gastroenterology, University College Hospital, London WCIE 6AU \\ M Sarner, MD, consultant physician \\ P B Boulos, Ms, consultant surgeon}

Department of Clinical Tropical Medicine, Hospital for Tropical Diseases, London NW1 M G Brook, MD, senior registrar in infectious diseases G C Cook, DSC, senior lecturer in clinical tropical medicine

Correspondence to: Dr Wilson.

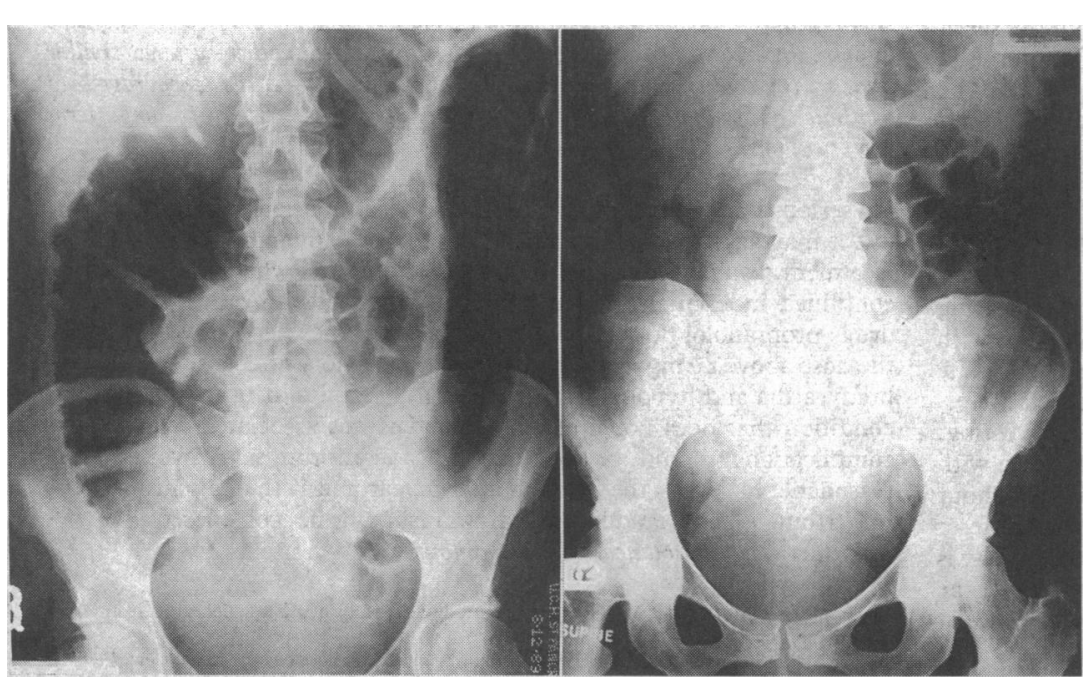

Abdominal radiographs of case 2. Left: on admission, showing gross colonic dilatation. Right: four week after admission, after intensive treatment and intravenous ciprofloxacin showing resolution of dilatation
Toxic megacolon is a rare complication of shigellosis (bacillary dysentery) but it occurs with acute infections caused by Salmonella spp, Campylobacter jejuni, and Yersinia enterocolitica.$^{1-4} \mathrm{~A}$ correct diagnosis is essential because treatment with corticosteroids can cause serious, potentially fatal complications. Furthermore, an unnecessary colonic resection may be performed inadvertently. The following two cases show that toxic megacolon can result from shigellosis in travellers even when faecal culture is negative.

\section{Case reports}

CASE 1

A 43 year old man returned from a two week stay in Accra, Ghana, where he had been admitted to hospital with a five day history of severe diarrhoea of acute onset. Chloramphenicol and trimethoprim had been ineffective. On admission he was febrile, dehydrated, and had watery stools (>six daily). Sigmoidoscopy showed an inflamed friable rectal mucosa with copious pus; immediate microscropic examination of stools for amoebic trophozoites was negative. A plain radiograph showed a grossly dilated colon. Three faecal cultures did not show Salmonella, Shigella, Campylobacter, or Vibrio spp and the toxin of Clostridium difficile was not detected. Ova, cysts (including Cryptosporidium spp), and parasites were not found. Four blood cultures were negative. Antibodies to Entamoeba histolytica were not found by immunofluorescence. Malaria parasites were not present in peripheral blood.

Intravenous rehydration and trimethoprim for three days failed to produce an improvement. A diagnosis of toxic megacolon seemed increasingly likely, but the clinical features did not seem consistent with a colitis; the colonic mucosa was inflamed but not ulcerated or bleeding. The patient remained toxic with a distended and tender abdomen. Serial radiographs showed increasing colonic dilatation.

Because of uncertainty about the diagnosis surgical decompression was considered necessary. At lapar- otomy the colon was grossly dilated and its wall oedematous and inflamed. The peritoneal cavity contained cloudy fluid. Endoscopy during the operation did not show any convincing mucosal changes and colonic resection was not considered. The colon was deflated both distally (with a suction tube through the rectum) and proximally (by an enterotomy which was fashioned as a defunctioning ileostomy). The procedure was carried out under antibiotic cover with cefuroxime and metronidazole.

Postoperatively he remained feverish, further cultures were obtained, and ampicillin was started. Although cultures of faeces, peritoneal fluid, and bone marrow were negative, a single blood culture produced a Gram negative bacillus, later identified as Shigella flexneri serotype 2a (Division of Enteric Pathogens, Colindale). It was sensitive to ciprofloxacin and resistant to ampicillin, trimethoprim, and chloramphenicol. Ciprofloxacin (200 mg intravenously 12 hourly) was started. His diarrhoea improved and by the fourth day he did not have a fever. He was discharged 15 days after surgery and readmitted for closure of his ileostomy. Subsequent recovery was uneventful.

\section{CASE 2}

A 40 year old woman was transferred from another hospital with a three week history of a severe dysenteric illness acquired in Morocco. Shigella flexneri type 4a had been isolated from a faecal sample, but blood cultures had been negative. Treatment was with trimethoprim, to which the organism was sensitive in vitro, and an opiate analgesic. On examination she was unwell, dehydrated, febrile $\left(38^{\circ} \mathrm{C}\right)$, and tender on abdominal palpation. An abdominal radiograph (figure) showed dilatation of the entire colon (maximum 10 $\mathrm{cm}$ ). Intensive medical management and ciprofloxacin (200 mg intravenously 12 hourly) but no corticosteroids produced a slow recovery over the next four weeks. A follow up radiograph confirmed resolution of the dilatation (figure). Rectal biopsy excluded non-infective inflammatory bowel disease.

\section{Discussion}

Despite full investigation an infective cause could not initially be found for the first patient's dysentery. Although reported in one patient with infection caused by Shigella sonnei, ${ }^{5}$ toxic megacolon is an unusual complication of bacillary dysentery, but it can develop in several acute bacterial infections. ${ }^{1-4}$ The use of corticosteroids was considered but could have proved disastrous had the underlying diagnosis not been discovered. Surgical intervention was unavoidable because of signs of impending perforation but, fortunately, careful judgment avoided unnecessary colonic resection. A lesser operation, which has been used before for toxic megacolon, ${ }^{6}$ was considered more appropriate. The strain of Shigella flexneri was resistant to all the antibiotics which had been used in the empirical treatment. In the second patient the pathogen was readily isolated but clinical deterioration occurred during treatment with oral trimethoprim; colonic distension raised the possibility of ulcerative colitis. 
In the first case the causative organism was isolated from a culture of the blood but not of the stool. Four patients with Shigella flexneri bacteraemia have been described in whom stool cultures were negative, ${ }^{15}$ and ileus or peritonitis are associated with bacteraemia. ${ }^{7}$ Bacteraemia, however, is rarely detected. Isolation of Shigella flexneri from the blood was recorded in only 34 patients reported from 1912 to $1962,{ }^{5}$ and in 38 of 1268 cases of dysentery caused by Shigella flexneri in Bangladesh. ${ }^{7}$ Shigella dysenteriae is more commonly invasive and was isolated from the blood of 36 of 451 cases in the same study. ${ }^{7}$ Bacteraemia caused by Shigella spp does not seem to be related to the severity of the illness and can be difficult to recognise, although it is apparently more common in children and infants. ${ }^{5}$

Failure to isolate the pathogen from the stool may be explained by intermittent excretion or by the reduced survival of Shigella spp if culture is delayed. ${ }^{8}$ Identification of all non-lactose fermenting colonies is often impracticable. Liquid selective media, such as Selenite F, may inhibit their growth, particularly that of Shigella flexneri. ${ }^{9}$ Antibiotic treatment before admission may also have affected excretion of the organism.

An infective cause of toxic dilatation of the colon was identified in 6 of 20 patients in the series of Schofield ${ }^{10}$; the remainder had Crohn's disease or ulcerative colitis. Although a positive culture is not an absolute indication of infective colitis, it can provide welcome support for continued conservative management of the patient. Negative cultures are potentially a much greater risk to a patient with severe infective colitis. The microbiologist must be consulted, and culture of the stool as well as blood should be performed urgently. Preferably, two to three samples should be taken before antibiotics are given. Standard methods of culture should be adequate for Shigella, Salmonella, and Campylobacter spp, but a selective medium for Yersinia spp should also be used. The possibility of pseudomembranous colitis should also be considered.

In retrospect, empirical antibiotic treatment was inappropriate in our cases; multiple antibiotic resistance is common in Shigella spp isolated from travellers returning to Britain. In 1982 resistance to ampicillin and chloramphenicol was present in $50 \%$ and to trimethoprim in $10 \%$ of strains submitted to the Division of Enteric Pathogens at Colindale. " More recently up to $50 \%$ of isolates have been trimethoprim resistant (Division of Enteric Pathogens at Colindale, personal communication). On current evidence a fluoroquinolone, such as ciprofloxacin, is the most appropriate empirical treatment of a patient with a probable severe infective colitis. ${ }^{12}$

If the initial cultures are negative and colonic symptoms and signs are not typical of ulcerative colitis or Crohn's disease strenuous efforts should be made to reach a bacterial diagnosis, and steroids should be used only with caution. A laparotomy may suggest the correct diagnosis first and allow decompression rather than resection of the colon.

We thank Dr A Hall for permission to describe case 1 and Dr B Rowe of the Division of Enteric Pathogens for help with bacterial identification.

1 Morduchowicz G, Huminer D, Siegman-Igra Y, Drucker M, Block CS, Pitlik SD. Shigella bacteremia in adults. A report of five cases and review of the literature. Arch Intern Med 1987;147:2034-7.

2 Thuluvath PJ, McKendrick MW. Salmonella and complications related to age - Sheffield experience. Qf Med 1988;67:497-503.

3 Stephenson TJ, Cotton DWK. Toxic megacolon complicating campylobacter colitis. BMF 1985;291:1242.

4 Stuart RC, Leahy AL, Cafferkey MT, Stephens RB. Yersinia enterocolitica infection and toxic megacolon. Br $\mathcal{F}$ Surg 1986;73:590.

5 Faucon R, Ducloux M. Septicemies a Shigella. A propos de deux nouvelles observations. Med Trop (Mars) 1964:24:537-45.

6 Turnbull RB, Hawk WA, Schofield P. Choice of operation for the toxic megacolon phase of non-specific colitis. Surg Clin North Am 1970;50: megacolon.

7 Struelens MJ, Patte D, Kabir I, Salam A, Nath SK, Butler T. Shigella septicaemia: prevalence, presentation, risk factors, and outcome. I Infect Dis 1985;152:784-90.

8 Cruikshank R, Swyer R. An outbreak of sonne dysentery. Lancet 1940;i: 803. 9 Taylor WI, Harris B. Isolation of shigellae II. Comparison of plating media and enrichment broths. Am $\mathcal{F}$ Clin Pathol 1965;44:476-9.

10 Schofield PF. Toxic dilatation and perforation in inflammatory bowel disease. Ann R Col Surg Engl 1982;64:318-9.

1 Rowe B, Threlfall EJ. Drug resistance in Gram-negative aerobic bacilli. Br Med Bull 1984;40:68-76.

12 Jewes LA. Antimicrobial therapy of non-typhi salmonella and shigella infection. F Antimicrob Chemother 1987:19.557-60.

(Accepted 1 November 1990

\section{ANY QUESTIONS}

\section{What is prickly heat? How is it diagnosed and what is the treatment?}

Prickly heat is a lay term to describe a rash that produces an annoying pricking sensation. Miliaria is the medical term.

The condition is due to the inability of sweat to escape from the skin either because the air is too humid or because the passage from the sweat duct to the skin is blocked or narrowed. It usually consists of small round macules, but these may coalesce to produce sheets of erythema. The flexures are the areas usually affected but so may be the face and trunk, especially in babies. The condition is precipitated by sweating, particularly in a humid atmosphere. So it is common in the humid tropics and in temperate climates when the weather is warm and humid, particularly when exercise provokes profuse sweating.

There is a condition affecting the feet known as juvenile plantar dermatosis. This is caused by occlusion of the ducts as a result of wearing shoes that do not permit the ready escape of sweat in conjunction with exercise, which causes a shearing stress on the skin rupturing the delicate sweat duct as it courses through the thick corneous layer of the sole.

Treatment consists of avoiding conditions that provoke sweating. Moving out of the hot sun, stopping exercise, and cooling the skin with cold water or ice may prove effective. The rash will subside within an hour or so. In the humid tropics this may prove impossible, so the sufferer should seek refuge in an air conditioned atmosphere where the humidity is low. If the condition becomes chronic the sufferer may have to leave the tropics because chronic miliaria may permanently damage the sweat glands. Topical remedies, such as lanolin or moisturising creams, may soften the sweat duct orifices and give some relief, but the precipitating factors must be avoided. Children with the foot disorder need to wear shoes with leather uppers and leather linings, which allow the sweat to escape.-ALAN B SHRANK, consultant dermatologist, Shrewsbury

A 40 year old woman has obtained complete relief from severe migraine attacks since starting propranolol $80 \mathrm{mg}$ daily. Her mother suffered simila attacks continuing into old age. Should the patient be encouraged to continue propranolol indefinitely or is there a risk of long term complications?

No serious long term complications have been reported with propranolol, but it may aggravate asthma or heart failure. Although the risks of this treatment would seem to be minimal, the question whether it should be continued indefinitely is debatable. In common with other $\beta$ blocking drugs propranolol does reduce drive. If it becomes necessary to increase the dose above $80 \mathrm{mg}$ daily there may be circulatory effects such as severe bradycardia and hypotension. In general indefinite drug treatment for a condition that does not threaten life and is not associated with serious complications should be avoided. As a compromise it would seem reasonable to review the position after a year and if the patient has been free from attacks gradual withdrawal should be considered.-BRYAN ASHWORTH, consultant neurologist, Edinburgh

Diamond S, Kudrow L, Stevens J, Shapiro DB. Long-term study of propranolol in the treatment of migraine. Headache 1982;22:268-71.

Steiner TJ, Joseph R. Practical experience of beta-blockade in migraine; a personal view. Postgrad Med f 1984;60(suppl 2):56-60. 